\title{
Plaque brachytherapy in iris and iridociliary melanoma: a systematic review of efficacy and complications
}

\author{
Saeed Karimi, MD 1,2, Amir Arabi, MD, MPH'.2, Toktam Shahraki, MD',2 \\ 'Ophthalmic Research Center, Research Institute for Ophthalmology and Vision Science, Shahid Beheshti University of Medical Sciences, \\ Tehran, Iran, ${ }^{2}$ Department of Ophthalmology, Torfeh Medical Center, Shahid Beheshti University of Medical Sciences, Tehran, Iran
}

\begin{abstract}
Purpose: To evaluate the efficacy and vision-threatening complication rate of plaque brachytherapy with iodine-125 $\left({ }^{125} \mathrm{I}\right)$, palladium-103 $\left({ }^{103} \mathrm{Pd}\right)$, and ruthenium-106 $\left({ }^{106} \mathrm{Ru}\right)$ for treatment of iris and iridociliary melanoma.

Material and methods: A literature review was done based on results yielded from searching PubMed, Embase, and Cochrane database, using following key words: iris melanoma, iridociliary melanoma, brachytherapy, iodine- 125 brachytherapy, palladium-103 brachytherapy, and ruthenium-106 brachytherapy. Initially, relationships between mean radiation dose to apex and local recurrence and complication rate were analyzed, and then, a comparison was performed between ${ }^{125} \mathrm{I},{ }^{103} \mathrm{Pd}$, and ${ }^{106} \mathrm{Ru}$ studies.

Results: Twelve retrospective and prospective studies were selected, with 491 patients treated primarily with plaque brachytherapy. The range of radiation dose to tumor apex were from 84 to 151.5 Gy. Ranges of mean and median of follow-up time were from 27 to 96 months. Local recurrence rate following brachytherapy ranged from 0 to $8 \%$. A decrease in the average study dose was not associated with an increased local recurrence or metastasis rate $(p=0.373$ and 0.195 , respectively); however, an increase in radiation dose was associated with higher radiationrelated cataract and glaucoma $(p<0.05)$. The rate of post-treatment glaucoma was higher in studies with ${ }^{125}$ I plaque brachytherapy $(p=0.004)$.

Conclusions: For brachytherapy of iris and iridociliary melanoma, in a range of 84 to $150 \mathrm{~Gy}$, an increase in radiation dose may increase the risk of complications, while the tumor control rate does not change.

J Contemp Brachytherapy 2021; 13, 1: 46-50 DOI: https://doi.org/10.5114/jcb.2021.103586
\end{abstract}

Key words: iris melanoma, iridociliary melanoma, brachytherapy, radiation dose, complications, ruthenium, iodine, palladium.

\section{Purpose}

Iris melanoma is believed to constitute less than $3 \%$ of uveal melanomas [1,2]. Considering a worldwide incidence of nearly 7,000 uveal melanomas per year [3], it seems that up to 200 cases of iris melanoma are diagnosed annually. The clinical signs, which are reported to be associated with malignant iris melanoma include significant stromal involvement with a thickness larger than $1 \mathrm{~mm}$, tumor vascularity, pigmentary glaucoma or ocular hypertension secondary to angle involvement, sectoral lens opacity, and presence of sentinel episcleral or iris vessels [4]. Even in the absence of these signs, a risk of malignant transformation is present for benign iris nevus with feathery margins, more than 4 clock-hour extension, ectropion uveae, and hyphema [5]. It has been reported that almost $5 \%$ of suspicious melanocytic lesions of iris increase in size in the first 5 years after diagnosis, and according to this low growth rate, the majority of iris melanocytic lesions have been primarily observed [6,7]. However, a metastatic rate of more than $10 \%$ have been recently reported for histologically proven iris melanomas [8]. It has made ophthalmologists more cautious in approaching suspicious melanocytic iris lesions [8,9]. Needle or aspiration cutter biopsy may be performed for suspicious iris tumors [10,11].

Iris and iridociliary melanoma is considered to be different from posterior uveal melanoma since it has been associated with favorable prognosis [12]. Management of iris and ciliary body melanoma includes plaque brachytherapy, proton beam radiation, iridectomy, and enucleation $[13,14,15]$. Following documentation of the tumor growth, or when there are clinical or histopathologic evidences of malignancy, surgery or radiotherapy may be performed.
Address for correspondence: Toktam Shahraki, MD, Ophthalmic Research Center, Research Institute for Ophthalmology and Vision Science, Shahid Beheshti University of Medical Sciences, No. 23, Paidarfard Ave., Boostan 9 Ave., Pasdaran St., 1666673111 Tehran, Iran, phone: +98-21-2258-5952, fax: +98-21-2259-0607, $\bowtie$ e-mail: toktam1372@gmail.com
Received: 11.08 .2020

Accepted: 06.11 .2020

Published: 18.02.2021 
Visual acuity, surgeon and patient's preference as well as location and features of the tumor determine treatment modality selected for each patient [5]. The main concern regarding radiotherapy for iris and iridociliary melanoma is the adverse effect of therapy on normal ocular tissues, especially crystalline lens and trabecular meshwork. On the other hand, surgical resection carries the risk of recurrence due to incomplete resection [14]. In certain conditions, a combined method, including both radiotherapy and surgery, may be used [16]. Within surgical methods, local resection by partial lamellar sclerouvectomy (PLSU) is a promising option in resection of a ciliary body melanoma. PLSU can be performed for tumors no greater than $12 \mathrm{~mm}$ in largest basal diameter, extending no more than $5 \mathrm{~mm}$ posteriorly to the equator [17].

${ }^{125} \mathrm{I},{ }^{103} \mathrm{Pd}$, and ${ }^{131} \mathrm{Cs}$ as low-energy seeds, and ${ }^{106} \mathrm{Ru}$ as $\beta$ emitter, have been used in radioactive plaques. In 1991 , the first case of iris melanoma treated with plaque brachytherapy was reported [18]. Following the report, a trend towards conservative plaque radiotherapy started, in which appropriate local control and proper conservation of healthy ocular tissues became the subject of interests.

The aim of the present study was to review the outcomes of brachytherapy of malignant iris and iridociliary melanoma with different radionuclides, and more importantly, to evaluate the rate of vision-threatening complications of this therapeutic method in different radiation doses and plaque types.

\section{Material and methods}

Our study consists of 12 peer-reviewed articles on the efficacy of brachytherapy for treatment of iris and iridociliary melanoma. PubMed, Embase, and Cochrane databases were searched using following keywords: "iris melanoma", "iridociliary melanoma", "brachytherapy", "iodine-125", "palladium-103", and "ruthenium-106". Following initial search, 280 papers were yielded. In order to extract appropriate articles, following inclusion criteria were applied: 1) available English language text, 2) performed on iris, ciliary body, or iridociliary malignant melanoma without involving posterior segment tumors, 3) data on mean or median of radiation dose to the apex of the tumor and 4) on mean or median of follow-up time, 5) percentage of local recurrence and systemic metastasis reported, 6) data on percentage of vision-threatening complications, including cataract and glaucoma following brachytherapy, 7) brachytherapy used as the primary single treatment. Accordingly, 12 reports were selected for this systematic review. Following parameters were obtained from each study: number of patients, type of the tumor (pure iris melanoma versus iridociliary or ciliary body melanoma), follow-up time (months), radiation dose to apex (Gy), local recurrence rate during follow-up period (\%), metastasis rate during follow-up period (\%), the rate of radiation-related cataract (\%), and the rate of radiation-related glaucoma or new onset of tumor-induced glaucoma following brachytherapy.

Linear regression was used to analyze the relationship between the mean apex dose and the recurrence or metastasis rate. Similarly, the relationship between the radiation dose and the vision-threatening complications was evaluated by regression analysis. Finally, as different radionuclides were used in the selected studies, a comparison of complications and recurrence rates was performed between ${ }^{125} \mathrm{I},{ }^{103} \mathrm{Pd}$, and ${ }^{106} \mathrm{Ru}$. In the analyses, each study was weighted according to the sample size. To compare the effectiveness and complication rates between three brachytherapy radionuclides, we pooled the data of each radionuclide studies, and the significance of the difference was analyzed by Fisher exact test. Statistical analysis was conducted using SPSS software. A regression coefficient was considered significant if the $p$-value was $<0.05$.

\section{Results}

A total of 12 studies (3 prospective and 9 retrospective) were included in this review (Table 1$)[18,19,20,21,22$, $23,24,25,26,27,28,29] .{ }^{125} \mathrm{I},{ }^{103} \mathrm{Pd}$, and ${ }^{106} \mathrm{Ru}$ plaques were used in 3,5, and 4 studies, respectively. The number of patients ranged from 11 to 144 . The mean and median of follow-up time differed from 27 to 96 months. Local recurrence rate following brachytherapy ranged from 0 to $8 \%$. Metastasis were reported in four studies, ranging from $1 \%$ to $4.5 \%$. The most common complication was radiation-induced cataract, ranging from $36 \%$ to $84 \%$. Another vision-threatening complication was glaucoma after brachytherapy occurring in $2 \%$ to $33 \%$ of patients.

Since two studies did not report a radiation dose to tumor apex, ten studies were involved in a linear regression analysis of the relationship between apex radiation dose and treatment failure and complications $[4,18,19,21,24,25,26,27,28,29]$. Within the above-described range, a decrease in radiation dose was not associated with an increased local failure or metastasis rate ( $p=0.373$ and 0.195 , respectively). On the other hand, an increase in radiation dose was significantly associated with a higher rate of post-treatment cataract $(p<0.05)$. Similarly, an increased radiation dose was observed to be associate with an increased rate of post-brachytherapy glaucoma $(p<0.05)$ (Figure 1$)$.

Respective to the type of radionuclide, it was observed that the prevalence of local recurrence and post-treatment glaucoma was more prominent in ${ }^{125} \mathrm{I}$ studies, and the difference was statistically significant $(p=0.019$ and 0.004 , respectively) (Table 2 ).

\section{Discussion}

Our review of 491 patients from 12 clinical studies is a large analysis on efficacy of plaque brachytherapy for iris and iridociliary melanoma. Plaque brachytherapy, independent of radionuclide, has shown prominent success in local control of iris and iridociliary melanoma. When combining the data of 12 retrospective and prospective studies, it seems that ${ }^{125} \mathrm{I}$ brachytherapy has revealed a lower rate of local tumor control compared to ${ }^{103} \mathrm{Pd}$ and ${ }^{106} \mathrm{Ru}$. According to our results, in a range of 80-150 Gy of apex radiation dose, a decrease in radiation dose was not associated with a lower tumor control. 


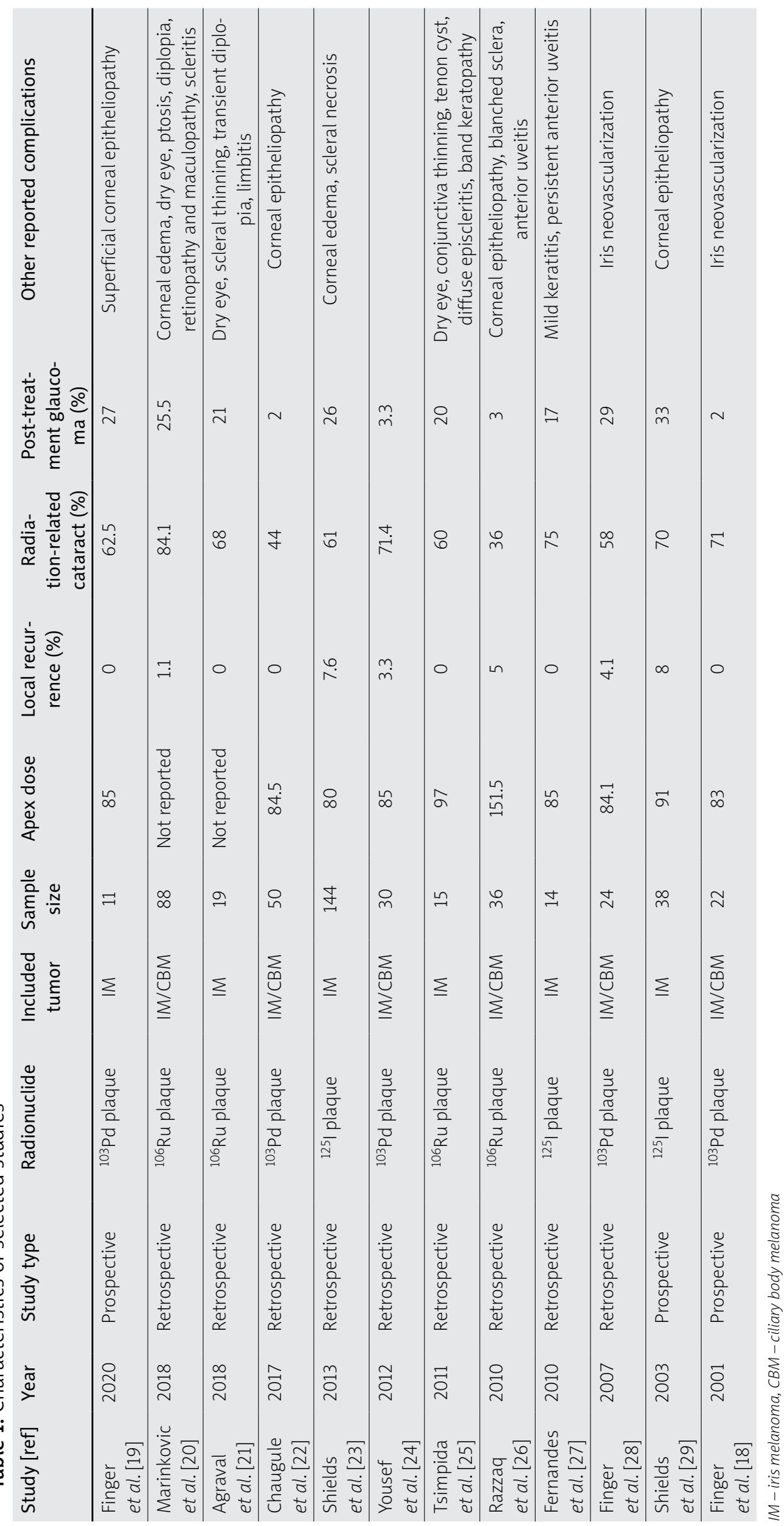


A

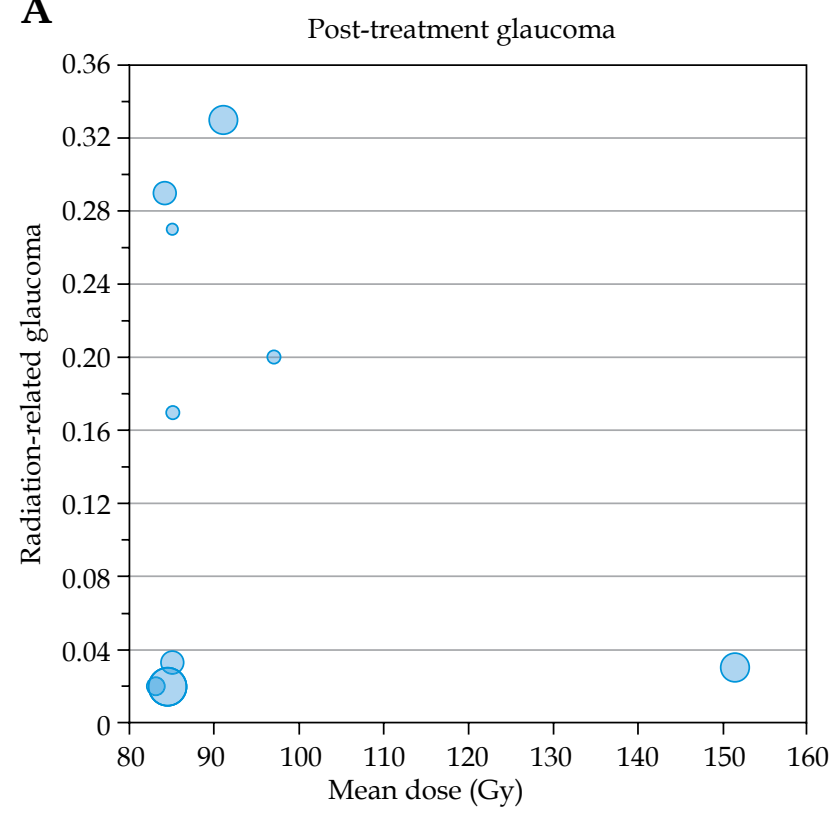

B

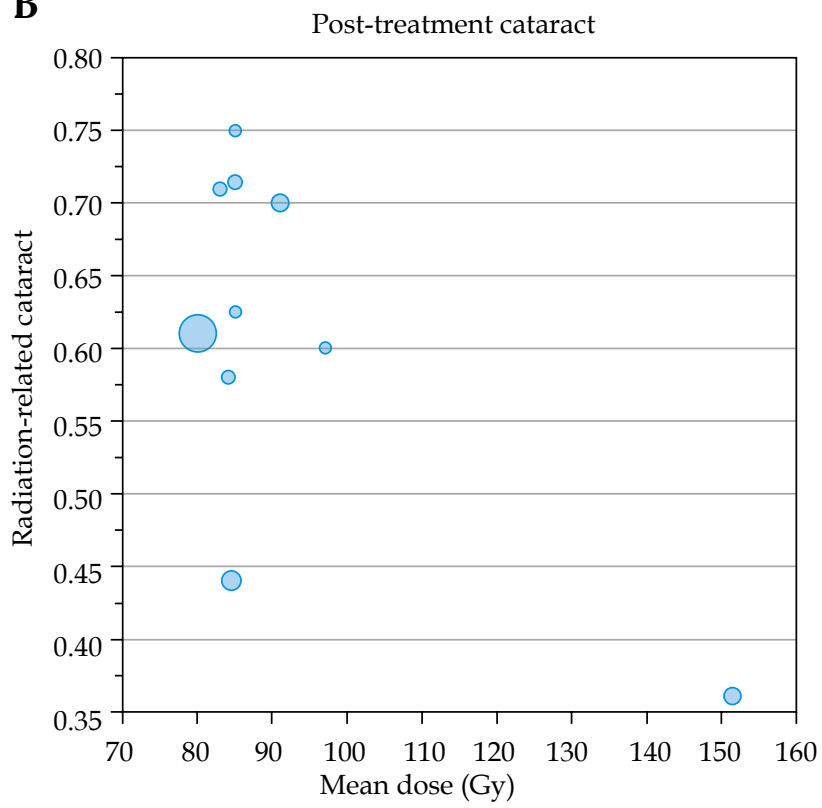

Fig. 1. Association between mean dose and radiation-related complications in published studies. Each bubble size is related to the patients included in the study

Table 2. A comparison between different radionuclides used for brachytherapy in iris and ciliary body melanoma, respective to recurrence and complication rates. Data of the studies is combined, and each study is weighted by the number of patients included

\begin{tabular}{|c|c|c|c|c|}
\hline Parameter & 125 | studies & ${ }^{106}$ Ru studies & ${ }^{103} \mathrm{Pd}$ studies & $P$-value \\
\hline Number of patients & 196 & 158 & 137 & \\
\hline Local recurrence (\%) & 6 & 1 & 1 & 0.019 \\
\hline Post-treatment cataract (\%) & 63 & 68 & 57 & 0.086 \\
\hline Post-treatment glaucoma (\%) & 26 & 19 & 8 & 0.004 \\
\hline
\end{tabular}

Transient anterior uveitis and superficial corneal epitheliopathy seem to be a universe complication following plaque brachytherapy for iris and iridociliary malignancies. However, in all reports, patients were successfully treated with topical medication. Even though there were concerns about scleral and corneal necrosis and melting, only one study reported scleral necrosis $(2 \%)$ following plaque removal [23]. Similarly, radiation retinopathy was rarely reported (4\% in one study only) [20].

Respective to the vision-threatening complications, radiation-related cataract was the most common adverse effect of plaque brachytherapy and the main cause of decreased vision following treatment. An increase in radiation dose was linked to a higher rate of post-treatment lens opacity. Cataract surgery in these patients was not associated with higher complications compared to non-radiation-related cataracts. There was no difference between ${ }^{125} \mathrm{I},{ }^{103} \mathrm{Pd}$, and ${ }^{106} \mathrm{Ru}$ plaques in the rate of post-treatment cataract.

Patients with iris and iridociliary melanomas may present with an increased intraocular pressure (IOP), which was secondary to pigment dispersion of the tumor, angle involvement, or iris neovascularization. After brachytherapy, some of these patients showed an improvement in ocular hypertension, as the regressed tumor may release less pigmentary particles and the tumor extension or iris vessels could relapse from anterior chamber angle. However, glaucoma might be an adverse effect of radiation therapy. Post-treatment glaucoma may be related to the formation of new vessels in anterior chamber, which ends in neovascular glaucoma, or it could be secondary to extensive pigment release from regressing tumor. We tried to include new onset glaucoma cases following plaque brachytherapy to analyze the rate of radiation-related glaucoma. According to our analysis, up to one third of iris and iridociliary melanoma patients could reveal ocular hypertension following plaque brachytherapy, and its probability increases with an increase in radiation dose. Also, the rate of post-treatment ocular hypertension seems to be more frequent in series treated with ${ }^{125} \mathrm{I}$. Both medical and surgical options were performed to treat radiation-related glaucoma in our review.

\section{Conclusions}

The present study involves a systematic review of 9 retrospective and 3 prospective studies. The main lim- 
itation of our review is the inclusion of retrospective studies, which did not allow to perform a formal meta-analysis, since there were no randomized trials and prospective studies were rare. However, published studies suggest that plaque brachytherapy is successful in local control and decrease the rate of metastasis of iris and iridociliary melanoma. All types of radionuclides used for plaques can cause cataract and glaucoma, as the two main vision-threatening complication of radiation. In the range of effective dose, a higher apical radiation dose may be associated with a higher rate of complications. Although analysis of combined data revealed a higher rate of glaucoma in ${ }^{125}$ I plaque therapy, this effect should be further investigated in randomized comparative studies.

\section{Disclosure}

The authors report no conflict of interest.

\section{References}

1. Jensen OA. Malignant melanomas of the human uvea. Recent follow-up of cases in Denmark, 1943-1952. Acta Ophthalmol 1970; 48: 1113-1128.

2. Shields CL, Shields JA, Materin M et al. Iris melanoma: risk factors for metastasis in 169 consecutive patients. Ophthalmology 2001; 108: 172-178.

3. Kivelä T. The epidemiological challenge of the most frequent eye cancer: retinoblastoma, an issue of birth and death. $\mathrm{Br}$ J Ophthalmol 2009; 93: 1129-1131.

4. Chaugule S, Finger P. Regression patterns of iris melanoma after Palladium-103 ((103)Pd) plaque brachytherapy. Ophthalmology 2017; 124: 1023-1030.

5. Shields CL, Kaliki S, Hutchinson A et al. Iris nevus growth into melanoma: analysis of 1611 consecutive eyes: the ABCDEF guide. Ophthalmology 2013; 120: 766-772.

6. Shields CL, Shields PW, Manalac J et al. Review of cystic and solid tumors of the iris. Oman J Ophthalmol 2013; 6: 159-164.

7. Territo C, Shields CL, Shields JA et al. Natural course of melanocytic tumors of the iris. Ophthalmology 1988; 95: 1251-1255.

8. Khan S, Finger PT, Yu GP et al. Clinical and pathologic characteristics of biopsy-proven iris melanoma: a multicenter international study. Arch Ophthalmol 2012; 130: 57-64.

9. Geisse LJ, Robertson DM. Iris melanomas. Am J Ophthalmol 1985; 99: 638-648.

10. Finger PT, Latkany P, Kurli M, Iacob C. The Finger iridectomy technique: small incision biopsy of anterior segment tumours. Br J Ophthalmol 2005; 89: 946-949.

11. Shields JA, Sanborn GE, Augsburger JJ. The differential diagnosis of malignant melanoma of the iris. A clinical study of 200 patients. Ophthalmology 1983; 90: 716-720.

12. Klauber S, Jensen PK, Prause JU, Kessing SV. Surgical treatment of iris and ciliary body melanoma: follow-up of a 25-year series of patients. Acta Ophthalmol 2012; 90: 122-126.

13. Damato B, Kacperek A, Chopra $\mathrm{M}$ et al. Proton beam radiotherapy of iris melanoma. Int J Radiat Oncol Biol Phys 2005; 63: 109-115.

14. Popovic M, Ahmed IIK, DiGiovanni J, Shields CL. Radiotherapeutic and surgical management of iris melanoma: A review. Surv Ophthalmol 2017; 62: 302-311.

15. Shields C, Grant Kels J, Shields JA. Melanoma of the eye: Revealing hidden secrets, one at a time. Clin Dermatol 2015; 33: 183-196.

16. Ramasubramanian A, Shields CL, Kytasty C et al. Resection of intraocular tumors (partial lamellar sclerouvectomy) in the pediatric age group. Ophthalmology 2012; 119: 2507-2513.
17. Shields JA, Shields CL, Shah P, Sivalingam V. Partial lamellar sclerouvectomy for ciliary body and choroidal tumors. Ophthalmology 1991; 98: 971-983.

18. Finger PT. Plaque radiation therapy for malignant melanoma of the iris and ciliary body. Am J Ophthalmol 2001; 132: 328-335.

19. Finger PT, Tomar AS, Chin KJ. Palladium-103 plaque therapy for multifocal iris melanoma: Radiation of the entire anterior segment of the eye. Eur J Ophthalmol 2020; 1120672120914235.

20. Marinkovic M, Horeweg N, Laman MS et al. Ruthenium-106 brachytherapy for iris and iridociliary melanomas. $\mathrm{Br} \mathrm{J} \mathrm{Oph-}$ thalmol 2018; 102: 1154-1159.

21. Agraval U, Sobti M, Russell HC et al. Use of Ruthenium-106 brachytherapy for iris melanoma: the Scottish experience. Br J Ophthalmol 2018; 102: 74-78.

22. Chaugule SS, Finger PT. Regression patterns of iris melanoma after Palladium-103 ((103)Pd) plaque brachytherapy. Ophthalmology 2017; 124: 1023-1030.

23. Shields CL, Shah SU, Bianciotto CG et al. Iris melanoma management with iodine- 125 plaque radiotherapy in 144 patients: impact of melanoma-related glaucoma on outcomes. Ophthalmology 2013; 120: 55-61.

24. Yousef YA, Finger PT. Lack of radiation maculopathy after palladium-103 plaque radiotherapy for iris melanoma. Int J Radiat Oncol Biol Phys 2012; 83: 1107-1112.

25. Tsimpida M, Hungerford J, Arora A, Cohen V. Plaque radiotherapy treatment with ruthenium-106 for iris malignant melanoma. Eye (Lond) 2011; 25: 1607-1611.

26. Razzaq L, Keunen JE, Schalij-Delfos NE et al. Ruthenium plaque radiation therapy for iris and iridociliary melanomas. Acta Ophthalmol 2012; 90: 291-296.

27. Fernandes BF, Krema H, Fulda E et al. Management of iris melanomas with 125Iodine plaque radiotherapy. Am J Ophthalmol 2010; 149: 70-76.

28. Finger PT, Reddy S, Chin K. High-frequency ultrasound characteristics of 24 iris and iridociliary melanomas: before and after plaque brachytherapy. Arch Ophthalmol 2007; 125: 1051-1058.

29. Shields CL, Naseripour M, Shields JA et al. Custom-designed plaque radiotherapy for nonresectable iris melanoma in 38 patients: tumor control and ocular complications. Am J Ophthalmol 2003; 135: 648-656. 